\title{
Synthesis, anti microbial screening and cytotoxic studies of some novel pyrazole analogs
}

\author{
K. Sony Jacob*, Swastika Ganguly \\ Department of Pharmaceutical Sciences and Technology, Birla Institute of Technology, Mesra, Ranchi -835215, Jharkhand, India.
}

\begin{tabular}{|c|c|}
\hline ARTICLE INFO & ABSTRACT \\
\hline $\begin{array}{l}\text { Article history: } \\
\text { Received on: } 29 / 07 / 2016 \\
\text { Revised on: } 21 / 08 / 2016 \\
\text { Accepted on: } 11 / 09 / 2016 \\
\text { Available online: } 29 / 11 / 2016\end{array}$ & $\begin{array}{l}\text { The objective of the study was to synthesize novel pyrazole analogs and evaluate their antimicrobial, } \\
\text { anthelmintic and cytotoxic activity. Novel } \\
\text { phenyldiazenyl)-1H-pyrazol-1-yl)ethanone derivatives were synthesized by conventional method using } \\
\text { potassium carbonate in DMF, under reflux condition. The antibacterial study was carried out against gram } \\
\text { positive and gram negative bacteria using ciprofloxacin and norfloxacin as standard drugs. Antifungal activity }\end{array}$ \\
\hline $\begin{array}{l}\text { Key words: } \\
\text { Pyrazole, anti-bacterial } \\
\text { activity, anti-fungal activity, } \\
\text { Dalton's lymphoma ascites } \\
\text { cells, Ehrlich ascites } \\
\text { carcinoma cells. }\end{array}$ & $\begin{array}{l}\text { (NCIM 1056). Anthelmentic activity assay was carried out on Indian earthworm, Pheretima posthuma. In vitro } \\
\text { cytotoxicity studies were also carried out using Dalton's lymphoma ascites cells (DLA) and Ehrlich ascites } \\
\text { carcinoma cells (EAC). Results of antibacterial study indicated that the compound } 22 \text { showed most promising } \\
\text { activity against both Gram-positive and Gram-negative organisms. Compound } 21 \text { and } 24 \text {, exhibited relatively } \\
\text { good inhibitory profile against Gram negative organism. In case of compounds } 25,27-30 \text { exhibited equally } \\
\text { potent antifungal activity as that of the standard drug fluconazole. Moreover, compound } 21-24,26 \text { showed } \\
\text { excellent activity against } C \text {. albicans and A.niger. The in vitro cytotoxicity results and anthelmintic reports } \\
\text { indicated compound } 22 \text { exhibited promising activity among the tested analogs. These newly synthesized } \\
\text { pyrazole analogs, especially } 21,22 \text { and } 24 \text { are better scaffolds to develop as broad spectrum chemotherapeutic } \\
\text { agents. }\end{array}$ \\
\hline
\end{tabular}

\section{INTRODUCTION}

Pyrazoles with several sites of alteration offer the flexibility to build up various structural analogs of biomedical concern. Compounds incorporating the pyrazolyl structural unit have shown significant biological activity, which include antiinflammatory and antipyretic (Rostom et al., 2009), antimicrobial (Javed and Hassan, 2013; Shukla et al., 2013 ), anthelmintic activity (Bruce, 2005 ; Wada et al., 2001), antiviral (Rashad et al., 2008 ; Mowbray et al., 2009 ) anticancer (Zhang et al., 2011), anticonvulsant (Farghaly et al., 2014 ), hypoglycemic (Das et al., 2008 ) carbonic anhydrase inhibitory (Balseven et al., 2013), and MAO inhibitory (Kumar et al., 2013) activity. It has

\section{* Corresponding Author}

Department of Pharmaceutical Sciences and Technology,

Birla Institute of Technology, Mesra, Ranchi -835215, Jharkhand, India.

Email: sonyivin@gmail.com been found that, 4-arylpyrazole derivatives exhibited unique role due to its antibacterial (Raimondi et al., 2012), analgesic (Oru et al., 2006), anticancer and DNA photocleavage activities (Kumar et al., 2014). A recent report showed that novel (E)-1-aryl-2-(3, 5dimethyl-4-(aryldiazenyl)-1H-pyrazol-1-yl) ethanones exhibited antimicrobial, antioxidant and UV-mediated DNA damage protective activity (Kaur et al., 2015). Moreover, pyrazole analogs exhibiting promising anticancer activities, especially on Ehrlich ascitic carcinoma cells (EAC) (Sunil et al., 2013) and on Dalton lymphoma acities cell lines (Ravula et al., 2016). Prompted from the above facts, we have synthesized some novel aryl azo pyrazoles using conventional methods using potassium carbonate in DMF under reflux condition . In order to explore biological potential of these novel pyrazole analogs, antibacterial, antifungal, anthelmintic and cytotoxicity activity of the synthesized compounds were carried out. 


\section{MATERIAL AND METHODS}

All the reagents used in this study were either of GR grade or of AR grade. They were purchased from commercial suppliers (Sigma Aldrich Company USA, Merck India Ltd,Himedia chemicals). The purity of the final compounds was checked by the TLC method. It was performed on Merck silica gel 60 F254 aluminium sheets using ethyl acetate: hexane (2:8) as eluents.

Iodine chamber and UV chamber used for the visualization of TLC spots. Melting points of compounds were determined on an optimelt automatic apparatus and were uncorrected.FT-IR spectra were recorded on SHIMADZU FT/IR 8400 and were reported in $\mathrm{cm}^{-1}{ }^{1}$ H-NMR spectra were recorded on Bruker advance digital spectrometer $400 \mathrm{MHz}$.Chemical shifts are expressed in $\delta$ values (ppm) relative to TMS as an internal standard, using $\mathrm{CDCl}_{3}$ solvent.

The mass spectra were recorded on a Jeol SX-106 instrument. Different steps involved in the synthesis of the final compounds are enumerated in Figure 1. Structures of the final compounds were confirmed using FT-IR, ${ }^{1} \mathrm{H}$ NMR and Mass,elemental analysis.

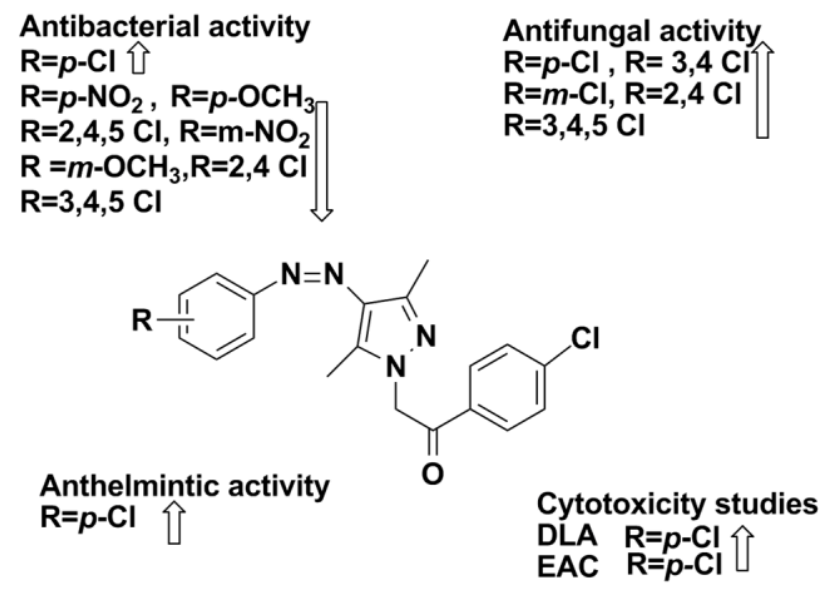

Fig. 1:

\section{EXPERIMENT}

Method of synthesis

General procedure for the synthesis of 3-(2-(substituited

phenyl)-hydrazono)-pentane-2,4-dione (1-10)

Intermediates are prepared by the reported procedures (Kaymakçıoglu et al., 2005).Appropriate substituited anilines $(0.01 \mathrm{~mol})$ was dissolved in a mixture of conc. $\mathrm{HCl}(1: 2)$ and water and cooled to $0{ }^{0} \mathrm{C}$ on ice bath.A cold aqueous solution of sodium nitrite $(0.02 \mathrm{~mol}, 1.38)$ was added.The cold diazonium salt solution was filterd into a cooled solution of acetyl acetone $(2.1 \mathrm{ml})$ in presence of sodium nitrite $(0.01 \mathrm{~mol}), 0.69)$ and sodium acetate $(0.05 \mathrm{~mol})$ in ethanol $/ \mathrm{methanol}(20 \mathrm{ml})$ and stirred room temperature for $2 \mathrm{hrs}$ and resulting solid was filtered, dried and purified by recrystallization from ethanol to afford the compound.
General procedure for the synthesis of (Z)-3,5-dimethyl-4(substituited phenyldiazenyl)-1H-pyrazole (11-20)

A mixture of 3-(2-(substituited phenyl)-hydrazono)pentane-2,4-dione (1g,0.003mole) and hydrazine hydrate (0.003mole) was heated under reflux in ethanol for 26hr. The reactions was moniterd by TLC using (chloroform: methanol, $8: 2$ ). The reaction mixture was cooled and the solids obtained were filterd and washed with water.The compounds were recrystallized from ethanol.

General procedure for the synthesis of (Z)-1-(4-chlorophenyl)-2(3,5-dimethyl-4-(substituited phenyldiazenyl)-1H-pyrazol-1yl)ethanone (21-30)

A mixture of (Z)-3,5-dimethyl-4-(substituited phenyldiazenyl)-1H-pyrazole $(0.004 \mathrm{~mol}), 4$ - chloro phenacyl bromide $(0.004 \mathrm{~mol})$ and anhydrous potassium carbonate $(50 \mathrm{mg})$ was heated under reflux in DMF for 20hrs.After completing the reaction, mixture was poured into ice cold water with stirring to yield the precipitate, which was further recrystallized from ethanol.

\section{Synthesis of (Z)-1-(4-chlorophenyl)-2-(4-((3-chlorophenyl) diazenyl)-3,5-dimethyl-1H-pyrazol-1-yl)ethanone (21)}

Yield: $80 \%$, m.p : $172^{\circ} \mathrm{C} \quad$,IR (KBr) $\left(\mathrm{cm}^{-1}\right): 1690(\mathrm{C}=\mathrm{O}$ str), 1553 (C=C str), 1592 (C=N str), ${ }^{1} \mathrm{H} \mathrm{NMR}\left(\mathrm{CDCl}_{3}, 400 \mathrm{MHz}\right)$ 7.29-7.99 (m,8H,Ar-H), $5.52\left(\mathrm{~s}, 2 \mathrm{H}, \mathrm{CH}_{2}\right), 2.53-2.59(\mathrm{~m}, 6 \mathrm{H}, 3,5$ $\left.\mathrm{CH}_{3}\right)$ EI-MS : 387.20(M+1) Anal. Calcd for $\mathrm{C}_{19} \mathrm{H}_{16}$ $\mathrm{Cl}_{2} \mathrm{~N}_{4} \mathrm{O}: \mathrm{C}, 58.93 ; \mathrm{H}, 4.16 ; \mathrm{N}, 14.47$.Found C,58.01; H,4.14; N, 14.07.

\section{Synthesis of ((Z)-1-(4-chlorophenyl)-2-(4-((4-chlorophenyl) diazenyl)-3,5-dimethyl-1H-pyrazol-1-yl)ethanone (22)}

Yield:82\%, m.p : $178{ }^{\circ} \mathrm{C}$,IR (KBr) $\left(\mathrm{cm}^{-1}\right): 1690(\mathrm{C}=\mathrm{O}$ str), 1550 ( $\mathrm{C}=\mathrm{C}$ str $), 1604$ ( $\mathrm{C}=\mathrm{N}$ str $),{ }^{1} \mathrm{H}$ NMR $\left(\mathrm{CDCl}_{3}, 400 \mathrm{MHz}\right)$ 7.28-7.99 (m,8H,Ar-H), $5.52\left(\mathrm{~s}, 2 \mathrm{H}, \mathrm{CH}_{2}\right), \quad 2.52-2.59(\mathrm{~m}, 6 \mathrm{H}, 3,5$ $\mathrm{CH}_{3}$ ) EI-MS : 387.26(M+1) Anal. Calcd for $\mathrm{C}_{19} \mathrm{H}_{16} \mathrm{Cl}_{2} \mathrm{~N}_{4} \mathrm{O}$ : C,58.93; H,4.16; N, 14.47.Found C,58.11; H,4.12; N, 14.17.

\section{Synthesis of (Z)-1-(4-chlorophenyl)-2-(4-((2,4-dichlorophenyl) diazenyl)-3,5-dimethyl-1H-pyrazol-1-yl)ethanone (23)}

Yield: $75 \%$, m.p : $186{ }^{\circ} \mathrm{C}$,IR (KBr) $\left(\mathrm{cm}^{-1}\right): 1690(\mathrm{C}=\mathrm{O}$ str), 1552 (C=C str), $1582(\mathrm{C}=\mathrm{N}$ str $),{ }^{1} \mathrm{H} \mathrm{NMR}\left(\mathrm{CDCl}_{3}, 400 \mathrm{MHz}\right)$ 7.32-8.03 (m,7H,Ar-H), $4.90\left(\mathrm{~s}, 2 \mathrm{H}, \mathrm{CH}_{2}\right), 3.04-3.07 \quad(\mathrm{~m}, 6 \mathrm{H}, 3,5$ $\left.\mathrm{CH}_{3}\right)$ EI-MS : 421.10(M+1) Anal. Calcd for $\mathrm{C}_{19} \mathrm{H}_{15} \mathrm{Cl}_{3} \mathrm{~N}_{4} \mathrm{O}$ : C,54.11; H,3.59; N, 13.29.Found C,54.41; H,3.24; N, 13.27

\section{Synthesis of (Z)-1-(4-chlorophenyl)-2-(4-((3,4-dichlorophenyl) diazenyl)-3,5-dimethyl-1H-pyrazol-1-yl)ethanone (24)}

Yield:70\%, m.p: $180{ }^{\circ} \mathrm{C}$,IR (KBr) $\left(\mathrm{cm}^{-1}\right): 1690(\mathrm{C}=\mathrm{O}$ str), 1550 ( $\mathrm{C}=\mathrm{C}$ str), 1599 (C=N str), ${ }^{1} \mathrm{H}$ NMR $\left(\mathrm{CDCl}_{3}, 400 \mathrm{MHz}\right)$ 7.33-8.03 (m,7H,Ar-H), $4.91 \quad\left(\mathrm{~s}, 2 \mathrm{H}, \mathrm{CH}_{2}\right), \quad 3.05-3.07 \quad(\mathrm{~m}, 6 \mathrm{H}, 3,5$ $\left.\mathrm{CH}_{3}\right)$ EI-MS : 421.70(M+1) Anal. Calcd for $\mathrm{C}_{19} \mathrm{H}_{15} \mathrm{Cl}_{3} \mathrm{~N}_{4} \mathrm{O}$ : C,54.11; H,3.59; N, 13.29.Found C,54.01; H,3.14; N, 13.07 
Synthesis of (Z)-1-(4-chlorophenyl)-2-(3,5-dimethyl-4-((2,4,5trichlorophenyl)diazenyl)-1H-pyrazol-1-yl)ethanone (25)

Yield:76\%, m.p : $170{ }^{\circ} \mathrm{C}, \mathrm{IR}(\mathrm{KBr})\left(\mathrm{cm}^{-1}\right): 1688(\mathrm{C}=\mathrm{O}$ str), 1552 ( $\mathrm{C}=\mathrm{C}$ str), 1594 ( $\mathrm{C}=\mathrm{N}$ str), ${ }^{1} \mathrm{H}$ NMR $\left(\mathrm{CDCl}_{3}, 400 \mathrm{MHz}\right)$ 7.28-7.63 (m,6H,Ar-H), $4.90\left(\mathrm{~s}, 2 \mathrm{H}, \mathrm{CH}_{2}\right), \quad 2.37-2.77(\mathrm{~m}, 6 \mathrm{H}, 3,5$ $\mathrm{CH}_{3}$ ) EI-MS : 456.10(M+2) Anal. Calcd for $\mathrm{C}_{19}$ $\mathrm{H}_{14} \mathrm{Cl}_{4} \quad \mathrm{~N}_{4} \mathrm{O}: \mathrm{C}, 50.03 ; \mathrm{H}, 3.09 ; \mathrm{N}, 12.28$. Found C,50.01; $\mathrm{H}, 3.14 ; \mathrm{N}, 12.07$

Synthesis of (Z)-1-(4-chlorophenyl)-2-(3,5-dimethyl-4-((3,4,5trichlorophenyl)diazenyl)-1H-pyrazol-1-yl)ethanone (26)

Yield:70\%, m.p : $174{ }^{\circ} \mathrm{C}$,IR (KBr) $\left(\mathrm{cm}^{-1}\right): 1686(\mathrm{C}=\mathrm{O}$ str), 1552 ( $\mathrm{C}=\mathrm{C}$ str), 1554 ( $\mathrm{C}=\mathrm{N}$ str), ${ }^{1} \mathrm{H}$ NMR $\left(\mathrm{CDCl}_{3}, 400 \mathrm{MHz}\right)$ 7.30-7.63 (m,6H,Ar-H), $4.99\left(\mathrm{~s}, 2 \mathrm{H}, \mathrm{CH}_{2}\right), \quad 2.34-2.77(\mathrm{~m}, 6 \mathrm{H}, 3,5$ $\mathrm{CH}_{3}$ ) EI-MS : 456.20(M+2) Anal. Calcd for $\mathrm{C}_{19} \mathrm{H}_{14}$ $\mathrm{Cl}_{4} \mathrm{~N}_{4} \mathrm{O}: \mathrm{C}, 50.03 ; \mathrm{H}, 3.09 ; \mathrm{N}, 12.28$.Found C,50.41; H,3.24; N, 12.17

Synthesis of (Z)-1-(4-chlorophenyl)-2-(3,5-dimethyl-4-((3nitrophenyl)diazenyl)-1H-pyrazol-1-yl)ethanone (27)

Yield:76\%, m.p : $188^{\circ} \mathrm{C}$,IR $(\mathrm{KBr})\left(\mathrm{cm}^{-1}\right): 1688(\mathrm{C}=\mathrm{O}$ str), 1553 ( $\mathrm{C}=\mathrm{C}$ str), 1594 ( $\mathrm{C}=\mathrm{N}$ str), ${ }^{1} \mathrm{H}$ NMR $\left(\mathrm{CDCl}_{3}, 400 \mathrm{MHz}\right)$ 7.51-8.33 (m,8H,Ar-H), $5.52\left(\mathrm{~s}, 2 \mathrm{H}, \mathrm{CH}_{2}\right), \quad 2.54-2.59(\mathrm{~m}, 6 \mathrm{H}, 3,5$ $\left.\mathrm{CH}_{3}\right)$ EI-MS : 397.21( $\left.\mathrm{M}^{+}\right)$Anal. Calcd for $\mathrm{C}_{19} \mathrm{H}_{16}$ $\mathrm{ClN}_{5} \mathrm{O}_{3}: \mathrm{C}, 57.36 ; \mathrm{H}, 4.05 ; \mathrm{N}, 17.60$.Found $\mathrm{C}, 57.41 ; \mathrm{H}, 4.11 ; \mathrm{N}$, 17.27

Synthesis of (Z)-1-(4-chlorophenyl)-2-(3,5-dimethyl-4-((4nitrophenyl)diazenyl)-1H-pyrazol-1-yl)ethanone(28)

Yield:70\%, m.p $180{ }^{\circ} \mathrm{C}$,IR (KBr) $\left(\mathrm{cm}^{-1}\right): 1690(\mathrm{C}=\mathrm{O}$ str), 1552 ( $\mathrm{C}=\mathrm{C}$ str), 1603 ( $\mathrm{C}=\mathrm{N}$ str), ${ }^{1} \mathrm{H}$ NMR $\left(\mathrm{CDCl}_{3}, 400 \mathrm{MHz}\right)$ 7.51-8.33 (m,8H,Ar-H), $5.52\left(\mathrm{~s}, 2 \mathrm{H}, \mathrm{CH}_{2}\right), 2.53-2.59(\mathrm{~m}, 6 \mathrm{H}, 3,5$ $\left.\mathrm{CH}_{3}\right)$ EI-MS : 397.81( $\left.\mathrm{M}^{+}\right)$Anal. Calcd for $\mathrm{C}_{19} \mathrm{H}_{16}$ $\mathrm{ClN}_{5} \mathrm{O}_{3}: \mathrm{C}, 57.36 ; \mathrm{H}, 4.05 ; \mathrm{N}, 17.60$.Found $\mathrm{C}, 57.01 ; \mathrm{H}, 4.14 ; \mathrm{N}$, 17.07

Synthesis of (Z)-1-(4-chlorophenyl)-2-(4-((3-methoxyphenyl) diazenyl)-3,5-dimethyl-1H-pyrazol-1-yl)ethanone (29)

Yield:78\%, m.p : $186{ }^{\circ} \mathrm{C}, \mathrm{IR}(\mathrm{KBr})\left(\mathrm{cm}^{-1}\right): 1690(\mathrm{C}=\mathrm{O}$ str), 1552 (C=C str), 1592 ( $\mathrm{C}=\mathrm{N}$ str), ${ }^{1} \mathrm{H}$ NMR $\left(\mathrm{CDCl}_{3}, 400 \mathrm{MHz}\right)$ 6.93-7.95 (m,8H,Ar-H), $5.27 \quad\left(\mathrm{~s}, 2 \mathrm{H}, \mathrm{CH}_{2}\right), \quad 2.49-2.55(\mathrm{~m}, 6 \mathrm{H}, 3,5$ $\left.\mathrm{CH}_{3}\right), 3.72-3.87\left(\mathrm{~d}, 3 \mathrm{H}, \mathrm{O}-\mathrm{CH}_{3}\right)$ EI-MS : $382.54\left(\mathrm{M}^{+}\right)$Anal. Calcd for $\mathrm{C}_{20} \mathrm{H}_{19} \mathrm{Cl} \mathrm{N}_{4} \mathrm{O}_{2}$ : C,62.74; H,5.00; N, 14.63.Found C,62.61; $\mathrm{H}, 5.01 ; \mathrm{N}, 14.56$

\section{Synthesis of (Z)-1-(4-chlorophenyl)-2-(4-((4-methoxyphenyl) diazenyl)-3,5-dimethyl-1H-pyrazol-1-yl)ethanone (30)}

Yield:70\%, m.p : $180^{\circ} \mathrm{C}$, IR (KBr) $\left(\mathrm{cm}^{-1}\right): 1690(\mathrm{C}=\mathrm{O}$ str), 1554 ( $\mathrm{C}=\mathrm{C}$ str), 1580 ( $\mathrm{C}=\mathrm{N}$ str), ${ }^{1} \mathrm{H}$ NMR $\left(\mathrm{CDCl}_{3}, 400 \mathrm{MHz}\right)$ 6.96-7.95 (m,8H,Ar-H), $5.47 \quad\left(\mathrm{~s}, 2 \mathrm{H}, \mathrm{CH}_{2}\right), \quad 2.50-2.55(\mathrm{~m}, 6 \mathrm{H}, 3,5$ $\left.\mathrm{CH}_{3}\right)$,3.73-3.87(d,3H,O-CH 3$)$ EI-MS : 382.84( $\left.{ }^{+}\right)$Anal. Calcd for $\mathrm{C}_{20} \mathrm{H}_{19} \mathrm{Cl} \mathrm{N}_{4} \mathrm{O}_{2}$ : C,62.74; H,5.00; N, 14.63.Found C,62.41; $\mathrm{H}, 5.11 ; \mathrm{N}, 14.66$

\section{MICROBIOLOGICAL SCREENING}

\section{Antibacterial screening}

All the synthesized compounds (21-30) were evaluated for their in vitro antimicrobial activity against six bacterial strains : Escherichia coli (MTCC 118),Pseudomonas aeruginosa (MTCC 647),Salmonella typhi (NCIM250), Klebsiella pneumonia (MTCC 3384),Bacillus subtilis (MTCC 121),Staphylococcus aureus (NCIM 2122) and fungal strains : Candida albicans (MTCC 227) and Aspergillus niger (NCIM 1056) which were procured from Institute of Microbial Technology, Chandigarh, India. The screening methods followed were as per National Committee for Clinical Laboratory Standards (NCCLS) protocol using Mueller Hinton Broth(Becton Dickinson, USA) (NCCL, 1993; NCCL,1992; Desai et al., 2013).The compounds which showed better activity while primary screening against microorganism at different concentrations of $1000,500,250 \mu \mathrm{g} / \mathrm{mL}$ was taken in to secondary screening. The second set of screening were performed with dilutions $100 \mu \mathrm{g} / \mathrm{mL}, 50 \mu \mathrm{g} / \mathrm{mL}, \quad 25 \mu \mathrm{g} / \mathrm{mL}, \quad 12.5 \mu \mathrm{g} / \mathrm{mL}$, $6.25 \mu \mathrm{g} / \mathrm{mL}, 3.125 \mu \mathrm{g} / \mathrm{mL}$. The minimum inhibitory concentration (MIC) of the synthesized compounds was determined by two dilution methods. The stock solution $(1000 \mu \mathrm{g} / \mathrm{mL})$ of the analogs were prepared in dimethyl sulfoxide (DMSO). The test tube with no compound but with equal volume of solvent DMSO (2\%) served as solvent control. One test tube with no compound and no vehicle but only with nutrient medium served as a negative control to ensure the growth property of the medium. Final concentration of bacterial strain was adjusted $10^{4} \mathrm{CFU} / \mathrm{mL}$ (Colony Forming Unit per milliliter). Nutrient medium, Mueller Hinton Broth was used to grow and dilute the test analogs suspension for bacteria. 2\% DMSO was taken as a vehicle to maintain the desired concentration of test analogs and standard drugs to perform the screening against microbial strains. The MIC values were measured after incubation at $35^{\circ} \mathrm{C}$ for a period of $24 \mathrm{hrs}$. The lowest concentration of the test compound that has completely inhibited the growth was reported as MIC.MIC values were also determined for standard drug Ciprofloxacin and Norfloxacin.

\section{Anti fungal screening}

The same compounds (21-30) were also screened for antifungal activity as primary screening against $C$. albicans and A.niger at various concentrations of $1000,500,250 \mu \mathrm{g} / \mathrm{mL}$. The compounds found to be active were taken into secondary screening with various concentrations of $100 \mu \mathrm{g} / \mathrm{mL}, 50 \mu \mathrm{g} / \mathrm{mL}$, $25 \mu \mathrm{g} / \mathrm{mL}, 12.5 \mu \mathrm{g} / \mathrm{mL}, 6.25 \mu \mathrm{g} / \mathrm{mL}, 3.125 \mu \mathrm{g} / \mathrm{mL}$. In case of antifungal screening, Sabouroud dextrose broth used as medium. The MIC values were recorded after incubation at $28 \pm 2{ }^{\circ} \mathrm{C}$ for $72 \mathrm{hrs}$.MIC values also determined for standard drug fluconazole.

\section{Anthelmintic activity studies}

The assay was executed on adult Indian earthworm, Pheretima posthuma due to its anatomical and physiological similarity with intestinal roundworm parasite of human beings (Vigar, 1984). Anthelmintic assay was performed as per the 
method given in literature (Vidyarthi,1967) with minor modifications. All the earthworms were collected from Divyayan Krishi Vigyan Kendra, Jharkhand, India .The earthworms, $P$. posthuma washed with normal saline $(0.5 \%)$ for about $30 \mathrm{~s}$ to remove all faecal matter, were used for anthelmintic study. Earthworms of 2-4 cm in length were used in this experiment and were divided into three groups of six each. All the compounds were dissolved in minimum quantity of $2 \% \mathrm{v} / \mathrm{v}$ Tween 80 . Before starting the experiments, all the compounds and standard drug solution were freshly prepared. All the synthesized compounds were subjected to study anthelmintic activity against earthworms at 5,10 and $20 \mathrm{mg} / \mathrm{mL}$ concentrations. The paralyzing and death times were noted and their mean was calculated for triplicate sets. Death time was recorded by placing earthworms in warm water and observed for stimulated movement, if the worm was alive. The synthesized compounds (21-30) were evaluated for their in vitro anthelmintic activity against Pheretima posthuma. Albendazole was used as a standard drug at a dose of $20 \mathrm{mg} / \mathrm{mL}$. The dose used for the newly synthesized compounds were 5,10 and $20 \mathrm{mg} / \mathrm{mL}$. The results are depicted in Table 2.

\section{Anticancer studies}

The test compounds were studied for short term in vitro cytotoxicity using Dalton's lymphoma ascites cells(DLA) or Ehrlich Ascites Carcinoma (EAC) cells . The studies were carried out in Amala Cancer Research Centre,Amala Nagar, Thrissur, Kerala.

\section{Trypan blue exclusion method:}

The tumour cells aspirated from the peritoneal cavity of tumour bearing mice were washed thrice with PBS or normal saline. Cell viability was determined by trypan blue exclusion method (Kuttan et al., 1985 ; Babu et al., 1995) Viable cell suspension $\left(1 \times 10^{6}\right.$ cells in $\left.0.1 \mathrm{~mL}\right)$ was added to tubes containing various concentrations of the test compounds and the volume was made up to $1 \mathrm{ml}$ using phosphate buffered saline (PBS).Control tube contained only cell suspension. These assay mixture were incubated for 3 hour at $37^{\circ} \mathrm{C}$. Further cell suspension was mixed with $0.1 \mathrm{ml}$ of $1 \%$ trypan blue and kept for 2-3 minutes and loaded on a haemocytometer. Dead cells take up the blue colour of trypan blue while live cells do not take up the dye. The number of stained and unstained cells were counted separately.

$$
\text { Percentage Cytotoxicity }=\frac{\text { No. of Dead cells }}{\text { No. of viable cells }+ \text { No. of dead cells }} \mathrm{X} 100
$$

\section{RESULTS AND DISCUSSION}

Various (Z)-1-(4-chlorophenyl)-2-(3,5-dimethyl-4 (substituited phenyldiazenyl)-1H-pyrazol-1-yl) ethanones were synthesized and characterized by analytical, FT-IR, ${ }^{1} \mathrm{H}$ NMR, Mass spectra techniques. The synthetic scheme of thesecompounds is represented in scheme 1 . As per literature, Intermediates were prepared by the reported procedures (Kaymakçioğlu et al., 2005). In addition, Kaur et al. 2015 have reported few antimicrobial, antioxidant and UV-mediated DNA damage protective photocleavage, anticancer activities of some novel (E)-1-aryl-2-(3, 5-dimethyl-4-(aryldiazenyl)-1H-pyrazol-1-yl)ethanones under solvent-free conditions. To synthesize target analogs, we decided to attach (Z)-3,5-dimethyl-4-(substituited phenyldiazenyl)-1Hpyrazole with 4-chloro phenacyl bromide by conventional method, using potassium carbonate in DMF under reflux condition. The products formed were (Z)-1-(4-chlorophenyl)-2-(4((3-chlorophenyl)diazenyl)-3,5-dimethyl -1H-pyrazol-1yl)ethanone (21), ((Z)-1-(4-chlorophenyl) -2-(4-((4chlorophenyl)diazenyl)-3,5-dimethyl-1H-pyrazol-1-yl) ethanone (22), (Z)-1-(4-chlorophenyl)-2-(4-((2,4-dichlorophenyl)diazenyl)3,5-dimethyl-1H-pyrazol-1-yl)ethanone (23), (Z)-1-(4chlorophenyl)-2-(4-((3,4-dichlorophenyl)diazenyl)-3,5-dimethyl1H-pyrazol-1-yl)ethanone (24), (Z)-1-(4-chlorophenyl)-2-(3,5dimethyl-4-((2,4,5-trichlorophenyl) diazenyl) -1H-pyrazol-1-yl) ethanone (25), (Z)-1-(4-chlorophenyl)-2-(3,5-dimethyl-4-((3,4,5trichlorophenyl)diazenyl)-1H-pyrazol-1-yl)ethanone (26). (Z)-1(4-chlorophenyl)-2-(3,5-dimethyl-4-((3-nitrophenyl)diazenyl)-1Hpyrazol-1-yl) ethanone (27), (Z)-1-(4-chlorophenyl)-2-(3,5dimethyl-4-((4-nitrophenyl)diazenyl)-1H-pyrazol-1-yl) ethanone (28), (Z)-1-(4-chlorophenyl)-2-(4-((3-methoxyphenyl)diazenyl)3,5-dimethyl-1H-pyrazol-1-yl)ethanone (29), (Z)-1-(4chlorophenyl)-2-(4-((4-methoxyphenyl)diazenyl) -3,5-dimethyl1H-pyrazol-1-yl)ethanone (30). The anti bacterial activity of synthesized compounds was assessed by comparison with ciprofloxacin and norfloxacin against some Gram-positive (S.aureus , B.subtilis) bacteria and Gram-negative (E.coli, P.aeruginosa , S.typhi , K.pneumoniae ) bacteria using serial dilution method and results were summarized in Table 1 . The anti bacterial results indicated that compounds 22 showed most promising activity against both Gram-positive and Gram-negative organism among the tested compounds. The compounds 21 and 24 showed relatively good inhibitory profile against Gram negative organism. In case of antifungal activities, compounds 21-24,26 showed excellent activity against $C$. albicans and A.niger .Moreover, compounds 25, 27-30 exhibited equally potent activity as standard drug fluconazole. Anthelmintic study results showed that, compound 22 exhibited better results among the screened series. The synthesized compounds were selected for the in vitro cell line studies using Dalton's lymphoma ascites cells(DLA) or Ehrlich Ascites Carcinoma (EAC) cells .The percentage cell death was calculated and were compared with the results of standard drug cyclophosphamide. The DLA cytotoxicity studies results depicted that $200 \mu \mathrm{g}$ of the compound 22 showed excellent cytotoxic activity (69\% cell death) than the standard drug of the same concentration ( $45 \%$ cell death) (Table 3). The EAC cytotoxicity studies also showed that $200 \mu \mathrm{g}$ of the compound 22 exibited promising cytotoxic activity (70\% cell death) than the standard drug cyclophosphamide of the same concentration $(48 \%$ cell death), whereas $100 \mu \mathrm{g}$ and $50 \mu \mathrm{g}$ of the same compound (22) showed better EAC cytotoxic effect $(46 \%$ cell death and $30 \%$ cell death) than the standard drug (37\% cell death and $28 \%$ cell death) of the same concentration (Table 4). 


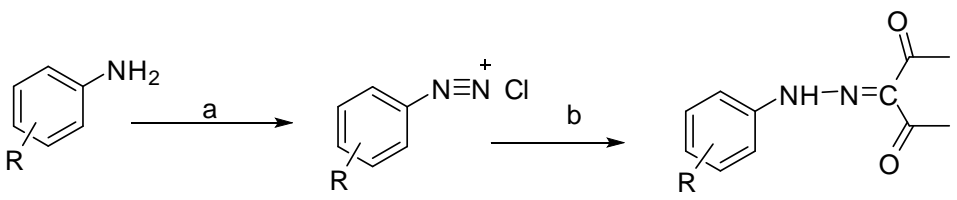

3-(2-substituted phenylhydrazono)pentane-2,4-dione

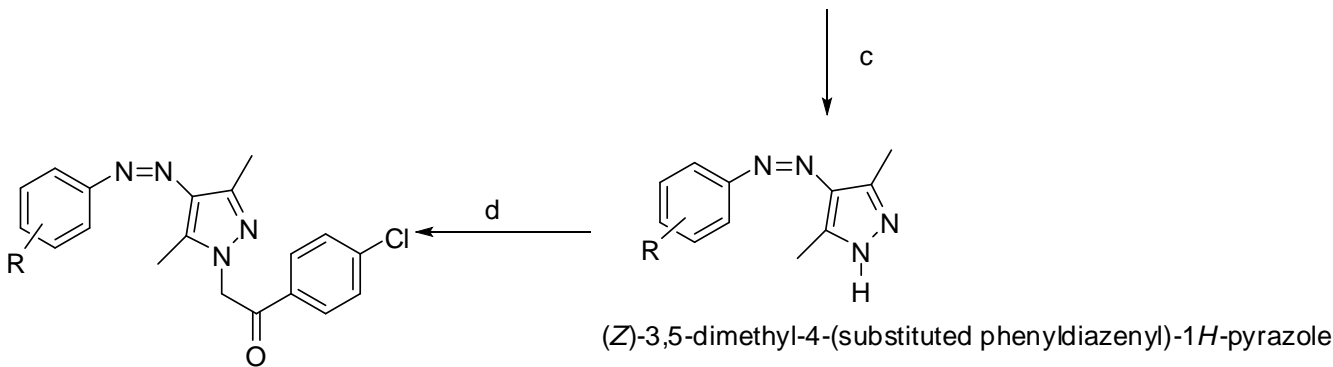

(Z)-1-(4-chlorophenyl)-2-(3,5-dimethyl-4-(substituted phenyldiazenyl)-1H-pyrazol-1-yl)ethanone

Reagents and conditions: a) $\mathrm{NaNO}_{2}, \mathrm{HCl}, 0-5^{\circ} \mathrm{C}$, stirring b)acetyl acetone, sodium acetate c)hydrazine hydrate,ethanol, reflux d) 4-chlorophenacylbromide,potassium carbonate,DMF, reflux

21) $m-\mathrm{Cl}, 22$ ) $\mathrm{R}=p-\mathrm{Cl}$, 23) $\mathrm{R}=2,4-\mathrm{Cl}, 24) \mathrm{R}=3,4-\mathrm{Cl}$, 25) $\mathrm{R}=2,4,5-\mathrm{Cl}, 26) 3,4,5-\mathrm{Cl}$, 27) $\mathrm{R}=m-\mathrm{NO}_{2}$, 28) $p-\mathrm{NO} 2$, 29) $\left.\mathrm{R}=m-\mathrm{OCH}_{3}, 30\right) \mathrm{R}=p-\mathrm{OCH}_{3}$

Scheme (1): Synthesis of (Z)-1-(4-chlorophenyl)-2-(3,5-dimethyl-4-(substituited phenyldiazenyl)-1H-pyrazol-1-yl)ethanone (21-30)

Table 1: Minimum Inhibitory concentration (MIC) of test compounds (21-30) against bacterial strains and fungal strains.

\begin{tabular}{|c|c|c|c|c|c|c|c|c|}
\hline \multirow{3}{*}{ Compounds } & \multicolumn{8}{|c|}{ Microorganisms } \\
\hline & \multicolumn{3}{|c|}{ Gram $+{ }^{\mathrm{ve}}$ bacteria } & \multicolumn{3}{|c|}{ Gram $-{ }^{\text {ve }}$ bacteria } & \multicolumn{2}{|c|}{ Fungal strains } \\
\hline & S.aureus & B.subtilis & E.coli & P.aeruginosa & S.typhi & K.pneumoniae & C.albicans & A.niger \\
\hline 21 & 6.25 & 6.25 & 6.25 & 6.25 & 3.12 & 3.12 & 3.12 & 3.12 \\
\hline 22 & 3.12 & 3.12 & 3.12 & 3.12 & 3.12 & 3.12 & 3.12 & 3.12 \\
\hline 23 & 100 & 50 & 50 & 50 & 50 & - & 3.12 & 3.12 \\
\hline 24 & 6.25 & 12.5 & 6.25 & 12.5 & 3.12 & 3.12 & 3.12 & 3.12 \\
\hline 25 & - & 100 & 50 & 100 & 12.5 & 100 & 12.5 & 12.5 \\
\hline 26 & 50 & 100 & - & - & - & 100 & 3.12 & 3.12 \\
\hline 27 & 100 & 50 & - & - & 100 & 100 & 12.5 & 12.5 \\
\hline 28 & 12.5 & 100 & 100 & 50 & - & - & 12.5 & 12.5 \\
\hline 29 & 50 & 12.5 & 50 & 25 & 50 & 50 & 12.5 & 12.5 \\
\hline 30 & 12.5 & - & - & 50 & 50 & - & 12.5 & 12.5 \\
\hline Ciprofloxacin & 0.78 & 1.56 & 0.39 & 0.78 & 0.78 & 0.78 & & \\
\hline Norfloxacin & 1.56 & 0.78 & 0.78 & 0.39 & 1.56 & 0.78 & & \\
\hline Fluconazole & & & & & & & 12.5 & 12.5 \\
\hline
\end{tabular}

Table 2: Anthelmintic activity of test compounds $(21-30)$ against Pheretima Posthuma.

\begin{tabular}{|c|c|c|c|c|c|c|}
\hline \multirow{3}{*}{ Compounds } & \multicolumn{3}{|c|}{ Mean time taken for paralysis } & \multicolumn{3}{|c|}{ and death of organism } \\
\hline & \multicolumn{3}{|c|}{ Paralysis time (Min) } & \multicolumn{3}{|c|}{ Death time (Min) } \\
\hline & $5 \mathrm{mg}$ & $10 \mathrm{mg}$ & $20 \mathrm{mg}$ & $5 \mathrm{mg}$ & $10 \mathrm{mg}$ & $20 \mathrm{mg}$ \\
\hline 21 & $16.34 \pm 1.10$ & $13.45 \pm 0.48$ & $08.32 \pm 0.52$ & $20.24 \pm 1.15$ & $16.14 \pm 0.35$ & $10.02 \pm 0.57$ \\
\hline 22 & $14.20 \pm 1.15$ & $10.11 \pm 0.61$ & $07.45 \pm 0.85$ & $16.02 \pm 1.22$ & $13.11 \pm 0.79$ & $08.10 \pm 0.61$ \\
\hline 23 & $15.33 \pm 0.78$ & $10.12 \pm 0.64$ & $08.35 \pm 0.67$ & $18.22 \pm 0.63$ & $13.24 \pm 0.59$ & $10.21 \pm 0.59$ \\
\hline 24 & $26.12 \pm 1.16$ & $16.10 \pm 0.78$ & $10.34 \pm 0.77$ & $29.23 \pm 1.22$ & $20.35 \pm 0.72$ & $11.20 \pm 0.64$ \\
\hline 25 & $28.10 \pm 1.18$ & $18.12 \pm 0.56$ & $09.21 \pm 0.82$ & $32.04 \pm 1.32$ & $22.31 \pm 0.71$ & $11.22 \pm 0.65$ \\
\hline 26 & $17.40 \pm 0.59$ & $14.32 \pm 0.48$ & $09.24 \pm 0.48$ & $20.10 \pm 0.44$ & $16.32 \pm 0.68$ & $12.29 \pm 0.47$ \\
\hline 27 & $18.11 \pm 0.87$ & $10.35 \pm 0.58$ & $08.34 \pm 0.87$ & $22.24 \pm 1.11$ & $14.20 \pm 0.46$ & $10.12 \pm 0.79$ \\
\hline 28 & $18.80 \pm 0.48$ & $9.45 \pm 0.54$ & $08.23 \pm 0.33$ & $20.23 \pm 0.57$ & $11.20 \pm 0.46$ & $10.25 \pm 0.42$ \\
\hline 29 & $25.08 \pm 1.30$ & $13.25 \pm 0.44$ & $08.19 \pm 0.32$ & $28.40 \pm 1.21$ & $18.20 \pm 0.57$ & $10.30 \pm 0.69$ \\
\hline 30 & $28.22 \pm 1.23$ & $14.20 \pm 0.62$ & $08.10 \pm 0.67$ & $30.21 \pm 1.24$ & $18.20 \pm 0.59$ & $10.24 \pm 0.49$ \\
\hline Albendazole & & & $05.32 \pm 0.72$ & & & $08.35 \pm 0.54$ \\
\hline
\end{tabular}


Table 3: Percentage cell death -Tested analogs vs .Standard drug (compounds 21-30).

\begin{tabular}{|c|c|c|c|c|c|c|c|c|c|c|c|}
\hline \multicolumn{11}{|c|}{ Percentage cell death -DLA (\%) } & \multirow[t]{2}{*}{ Standard cyclophosphamide (\%) } \\
\hline Conc. $\mu \mathrm{g} / \mathrm{mL}$ & 21 & 22 & 23 & 24 & 25 & 26 & 27 & 28 & 29 & 30 & \\
\hline $200 \mu \mathrm{g}$ & 25 & 69 & 10 & 2 & 24 & 39 & 6 & 29 & 45 & 27 & 45 \\
\hline $100 \mu \mathrm{g}$ & 10 & 30 & 5 & - & 17 & 22 & 0 & 16 & 30 & 19 & 40 \\
\hline $50 \mu \mathrm{g}$ & 2 & 24 & - & - & 6 & 8 & - & 3 & 18 & 11 & 26 \\
\hline $20 \mu \mathrm{g}$ & - & 10 & - & - & - & - & - & - & 6 & 7 & 15 \\
\hline $10 \mu \mathrm{g}$ & & 2 & 2 & - & 2 & - & - & 2 & - & - & 13 \\
\hline
\end{tabular}

Table 4: Percentage cell death -Tested analogs vs .Standard drug (21-30).

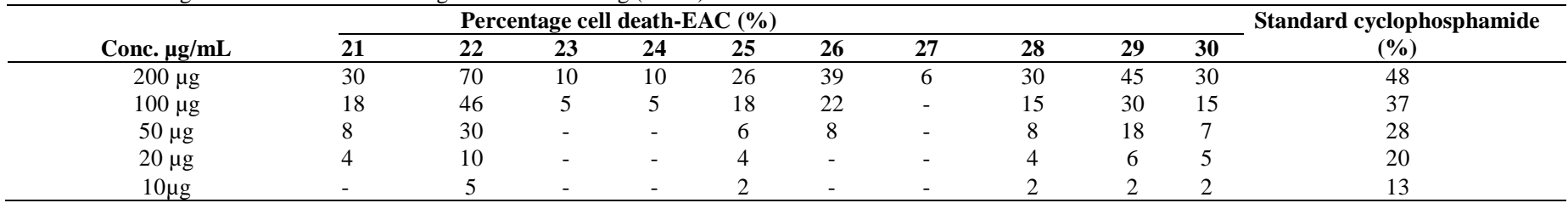

\section{Structure Activity Relationship (SAR)studies}

From these preliminary screening studies, it was found that the presence of halogens in different positions of the aromatic ring of these novel pyrazole analogs assured their activity. The overall effects of substitution pattern on the different biological activities are summarized in Fig 1.

It was interesting to notice that test compound 22 with chloro substitution in para position led to a significant increase in antibacterial activity and anthelmintic and cytotoxic activities.However, presence of halogen substituted in the different positions in the aromatic ring played a substantial increase in the antifungal activities.This indicates that the electron withdrawing groups played a considerable role in a substantial increase in the in vitro screening results.

\section{CONCLUSION}

In conclusion, a series of (Z)-1-(4-chlorophenyl)-2-(3,5dimethyl-4- (substituited phenyldiazenyl)-1H-pyrazol-1-yl) ethanones (21-30) has been synthesized using conventional method using potassium carbonate in DMF, under reflux condition and their structures were established by IR,NMR ,Mass and elemental analysis. The results of antibacterial results revealed that compounds 22 showed most promising activity against both Grampositive and Gram-negative organism among the tested compounds. The compounds 21 and 24 showed relatively good inhibitory profile against Gram negative organism compared to the standard drug ciprofloxacin and Norfloxacin.In case of antifungal activities , compounds 21-24,26 showed excellent activity against $C$. albicans and A.niger. Moreover, compounds 25,27-30 exhibited equally potent activity as standard drug fluconazole. However, anthelmintic study results showed that, compound 22 exhibited better results among the screened series. Furthermore, in vitro DLA cytotoxicity studies results depicted that $200 \mu \mathrm{g}$ of the compound 22 showed excellent cytotoxic activity (69\% cell death) than the standard drug of the same concentration ( $45 \%$ cell death) and EAC cytotoxicity studies also showed that $200 \mu \mathrm{g}$ of the compound 22 exibited promising cytotoxic activity $(70 \%$ cell death) than the standard drug cyclophosphamide of the same concentration (48\% cell death),whereas $100 \mu \mathrm{g}$ and $50 \mu \mathrm{g}$ of the same compound (22) showed better EACcytotoxic effect $(46 \%$ cell death and $30 \%$ cell death) than the standard drug (37\% cell death and $28 \%$ cell death) of the same concentration . Findings from this study revealed that, these newly synthesized pyrazole analogs especially $21,22,24$ could serve as better scaffolds to develop as broad spectrum chemotherapeutic agents.

\section{CONFLICT OF INTEREST}

The authors confirm that this article content has no conflict of interest.

\section{ACKNOWLEDGMENTS}

The authors acknowledge Department of Pharmaceutical Sciences and Technology, BIT, Mesra, Amala Cancer Research Centre, Thrissur, Kerala for providing necessary facilities to carry out this study. The authors also acknowledge IIRBS, M.G. University, Kerala and Dr. Reddy's Institute of Life Sciences, Hyderabad for spectral data.

Financial support and sponsorship: The authors acknowledge University Grants Commission (UGC) for financial support in the form of Maulana Azad fellowship (MANF-2012-13-CHR-KER13883).

Conflict of Interests: There are no conflicts of interest.

\section{REFERENCES}

Babu TD, Kuttan G, Padikkala J. Cytotoxic and anti-tumour properties of certain taxa of Umbelliferae with special reference to Centella asiatica (L.) Urban. J. Ethnopharmacol 1995 ; 48 :53-57.

Balseven H, Isgor MM, Mert S, Alım Z, Beydemir S, Ok S, Kasımog ullar1 R. Facile synthesis and characterization of novel pyrazolesulfonamides and their inhibition effects on human carbonic anhydrase isoenzymes. Bioorg Med Chem, 2013;21:21-27.

Bruce, C . 2005. Anthelmintic compositions. U.S.11/318,107. 
Das N, Verma A, Shrivastava PK, Shrivastava SK. Synthesis and biological evaluation of some new aryl pyrazol-3-one derivatives as potential hypoglycemic agents. Indian J. Chem. 2008 ; 47B : 1555-1558.

Desai NC, Rajpara KM, Joshi VV, Vaghani HV, Satodiya HM. Synthesis, characterization and antimicrobial screening of hybrid molecules containing quinoline, pyrimidine and morpholine analogues. J. Chem. Sci $2013 ; 125: 321-333$.

Farghaly AR, Esmail S, Abdel-Zaher A, Abdel-Hafez A, ElKashef H. Synthesis and anticonvulsant activity of some new pyrazolo[3 4-b]pyrazines and related heterocycles. Bioorg Med Chem, 2014;22: 2166-2175.

Javed MASA, Hassan MH. Synthesis and antimicrobial activity of pyrazoline and pyrazole analogues containing quinoline moiety. Indian J Chem 2013; B 52:1493-1499.

Kaymakçığlu BK, Rollas S, Körceğez E, Arıcıŏlu F. Synthesis and biological evaluation of new N-substituted-N'-(3,5-di/1,3,5trimethylpyrazole-4-yl) thiourea/urea derivatives. Eur. J. Pharm. Sci 2005 ; $26: 97-103$.

Kuttan R, Bhanumathy P, Nirmala K, George MC. Potential anti-cancer activity of turmeric. Cancer Lett $1985 ; 29: 197-202$.

Kumar A, Jain S, Parle M, Jain N, Kumar P. 3-aryl-1-phenyl1h-pyrazole derivatives as new multitarget directed ligands for the treatment of Alzheimer's disease with acetylcholinesterase and Monoamine oxidase inhibitory properties. EXCLI Journal, 2013; 12: 1030-1042.

Kumar V, Kaur K, Karelia DN, Beniwal V, Gupta GK, Sharma AK,Gupta AK. Synthesis and biological evaluation of some2-(3,5dimethyl-1H-pyrazol-1-yl)-1-arylethanones: bacterial, DNA photo cleavage, and anticancer activities. Eur J Med Chem 2014; 81:267-276. Sunil D, Isloor AM, Shetty P, Nayak PG , Pai KSR. 2013. In vivo anticancer and histopathology studies of Schiff bases on Ehrlich ascitic carcinoma cells: 1st cancer update. Arabian J. Chem 2013 ; 6 :25-33.

Kaur K, Kumar V, Beniwal V, Kumar V, Aneja KR, Sharma V, Jaglan S. Novel (E)-1-aryl-2-(3, 5-dimethyl-4-(aryldiazenyl)-1H-pyrazol1-yl) ethanones: solvent-free synthesis and antimicrobial, antioxidant and UV-mediated DNA damage protective activity.Med Chem Res, 2015; 24:4023-4036

Mowbray CE, Burt C, Corbau R, Gayton S, Hawes M, Perros M, Tran I, Price DA, Quinton FJ, Selby MD, Stupple PA, Webster R, Wood A. Pyrazole NNRTIs 4: Selection of UK-453 061 (lersivirine) as a development Candidate. Bioorg. Med. Chem. Lett, 2009; 19 : 5857-5860.

National Committee for Clinical Laboratory Standards, Methods for Dilution Antimicrobial Susceptibility Tests for Bacteria that Grow Aerobically Approved Standard, third ed.; NCCLS Publication M7-A3 : Villanova, PA, 1993.

National Committee for Clinical Laboratory Standards, Reference Method for Broth Dilution Antifungal Testing of Yeasts, Proposed Standard NCCLS Document M27-P, Wayne ,1992.

Oru EE, Koyigit-Kaymakioglu B, Oral B, Altunbas-Toklu HZ, Kabasakal L, Rollas S. Synthesis of some novel azo derivatives of 3,5dimethyl-1-(2-hydroxyethyl)pyrazole as potent analgesic agents. Arch Pharm Chem Life Sci, 2006 ; 339: 267-272.
Rashad AE, Hegab MI, Abdel-Megeid RE, Micky JA, AbdelMageid FME.Synthesis and antiviral evaluation of some new pyrazole and fused pyrazolopyrimidine derivatives. Bioorg Med Chem, 2008; 16: 7102-7106.

Rostom SAF, El-Ashmawy IM, Abd El Razik HA, Badr MH, Ashour HMA. Design and synthesis of some thiazolyl and thiadiazolyl derivatives of antipyrine as potential non-acidic anti-inflammatory analgesic and antimicrobial agents. Bioorg Med Chem, 2009; 17: 882895.

Raimondi MV, Maggio B, Raffa D, Plescia F, Cascioferro S, CancemiG, Schillaci D, Cusimano MG, Vitale M, Daidone G. Synthesis and anti-staphylococcal activity of new 4-diazopyrazole derivatives. Eur J Med Chem, 2012 ; 58: 64-71.

Ravula P, Vamaraju HB, Paturi M, Kolli S. Design, synthesis, in silico toxicity prediction, molecular docking, and evaluation of novel pyrazole derivatives as potential antiproliferative agents. EXCLI journal $2016 ; 15: 187-202$.

Shukla M, Seth DS, Kulshreshtha H . Green chemical approach to synthesize 1-(N-substituted aniline malonyl)-3,5-dimethyl-4-(3,4difluoro phenyl azo) pyrazoles and their antimicrobial evaluation. JOAC $2013 ; 2: 1484-1488$.

Sunil D, Isloor AM, Shetty P, Nayak PG, Pai KS. In vivo anticancer and histopathology studies of Schiff bases on Ehrlich ascitic carcinoma cells: 1st cancer update. Arabian J. Chem. 2013; $6: 25-33$.

Vigar Z. 1984. Atlas of medical parasitology. Singapore, P. G. Publishing House.

Vidyarthi RD.1967. A Text Book of Zoology. New Delhi, India: S. Chand and Co. Publishers.

Wada K., Gomibuchi T, Otsu Y, Shibuya K., Abe T, Andersch W, Harder A, Lösel P, Nihon Bayer Agrochem KK. 2001. Nematicidal pyrazoles. U.S. Patent $6,310,049$.

Zhang D, Wang G, Zhao G, Xu W, Huo L. Synthesis and cytotoxic activity of novel 3-(1H-indol-3-yl)-1H-pyrazole-5carbohydrazide derivatives. Eur J Med Chem , 2011; 46 : 5868-5877.

\section{How to cite this article:}

Jacob KS, Ganguly S. Synthesis, anti microbial screening and cytotoxic studies of some novel pyrazole analogs. J App Pharm Sci, 2016; 6 (11): 135-141. 